\title{
La plus belle histoire de l'École
}

Alain Boissinot et Luc Ferry, Robert Laffont, 2017, 486 p.

\section{Anne-Marie Bardi}

\section{OpenEdition}

\section{Journals}

Édition électronique

URL : https://journals.openedition.org/ries/6108

DOI : 10.4000/ries. 6108

ISSN : 2261-4265

Éditeur

France Education international

Édition imprimée

Date de publication : 30 avril 2018

Pagination : 29-31

ISBN : 978-2-85420-618-0

ISSN : 1254-4590

\section{Référence électronique}

Anne-Marie Bardi, «La plus belle histoire de l'École », Revue internationale d'éducation de Sèvres [En ligne], 77 | avril 2018, mis en ligne le 30 avril 2018, consulté le 25 juin 2021. URL : http:// journals.openedition.org/ries/6108; DOI : https://doi.org/10.4000/ries.6108

Ce document a été généré automatiquement le 25 juin 2021.

(c) Tous droits réservés 


\title{
La plus belle histoire de l'École
}

\author{
Alain Boissinot et Luc Ferry, Robert Laffont, 2017, 486 p.
}

\author{
Anne-Marie Bardi
}

1 Ce livre - qu'on le dévore ou qu'on le picore - ne nous raconte pas seulement une histoire de l'École: il enracine l'École dans l'Histoire, à travers l'étude de quatre grandes périodes intitulées: le monde gréco-romain (le modèle aristocratique), le Moyen Âge (l'école des clercs), de l'humanisme à la Révolution (vers une éducation nationale), l'époque contemporaine (l'âge de la démocratisation). Le lecteur attend donc avec impatience la cinquième partie " Et demain? ». Où l'entraînera-t-elle?

2 La plus belle histoire de l'École est écrite par un professeur de lettres supérieures ayant occupé de hautes responsabilités dans l'éducation nationale et par un philosophe ancien ministre, tous deux passionnés des questions d'éducation, ce qui donne à ce livre un agréable ton, parfois très personnel. Au fil des pages, nous relisons nos classiques, nous partageons la vivacité de certains débats, nous revisitons notre proche passé et nous en venons à regarder notre présent controversé avec une sévère bienveillance. D'un tel foisonnement, chacun pourra retenir ses propres leçons.

3 Les auteurs décrivent avec précision, pour chaque période, la cohérence entre l'École, son lieu et les organisations politiques et sociales de son temps. Ils nous proposent une plongée savante dans l'histoire de France et nous rappellent que, dans toute société, la vision du monde et la place donnée à l'homme sur terre et dans l'univers induisent les finalités de l'éducation, et donc ses modalités; ils signalent aussi, au-delà des cohérences, les forces à l'œuvre pour préparer de lentes transitions.

4 Car l'École peine à changer, même lorsque les temps changent, entraînant des tensions ou la création de solutions alternatives: l'Université s'étiole à la fin de l'Ancien Régime? Nos "grandes écoles» se créent en parallèle: Ponts et Chaussées, Mines, Génie, Vétérinaire, Polytechnique... Une originalité française qui dure depuis 250 ans ! Quant aux évolutions, elles demandent du temps: il faudra, par exemple, la contribution de tous les régimes du XIX siècle pour organiser peu à peu l'école primaire laïque et obligatoire.

5 Ce voyage dans l'Histoire nous fait également prendre de la distance avec notre présent, par exemple avec notre « heure de cours », avec les matières enseignées ou la 
place des filles. L'heure de cours est à peine centenaire! Au Moyen Âge, les écoles qui voient le jour dans les monastères et autour des cathédrales organisent des classes sans unité, où le maître s'adresse à chaque élève successivement. Il faut attendre le XVII ${ }^{\mathrm{e}}$ siècle pour qu'apparaisse "l'enseignement simultané ", par lequel le maître enseigne à un groupe relativement homogène. Jusqu'au $\mathrm{XX}^{\mathrm{e}}$ siècle, la classe est le lieu de l'exercice, de la correction des devoirs, de l'activité préparée en amont; les surveillants et les répétiteurs jouent un rôle important. Notre "classe inversée » en quelque sorte! Aujourd'hui, le cours est délivré par un professeur, dont l'activité semble primer sur celle des élèves, le travail de ces derniers étant reporté à l'extérieur, les « devoirs à la maison».

6 Les matières enseignées et leur hiérarchie ont également varié. En Grèce, on étudie les grands auteurs afin d'y puiser des principes de vie et de disposer de modèles. On apprend la musique qui initie au rythme, à l'harmonie et qui prépare de bons orateurs. La gymnastique vise à l'équilibre du corps, à sa beauté plastique et forme au combat, donc à la défense de la cité. Les futurs dirigeants sont de plus initiés à la rhétorique et à la sophistique, afin de savoir expliquer et convaincre. La formation assure la conformité à un modèle de civilisation, et donc la pérennité de celui-ci.

7 Au Moyen Âge, le latin s'affirme comme langue de culture. On enseigne d'abord la grammaire, la rhétorique et la logique ; viennent ensuite l'arithmétique, la musique, la géométrie et l'astronomie. Quelques centaines d'années plus tard, à la création de l'agrégation en 1766, celle-ci ne comporte que trois spécialités: grammaire, belleslettres et philosophie. L'agrégation de sciences ne voit le jour qu'en 1821. Peu à peu, les disciplines s'autonomisent et revendiquent la noblesse d'une agrégation. Mais en même temps que le nombre de disciplines s'accroît le nombre d'heures de cours des élèves !

Quant à l'éducation des filles... Certains se reconnaitront peut-être encore dans cette citation de Fénelon : « [1]a science des hommes comme celle des femmes doit se borner à s'instruire par rapport à leur fonction. La différence de leurs emplois doit faire celle de leurs études ». Si l'on instruit les filles c'est donc pour qu'elles éduquent mieux leurs enfants et qu'elles soient de meilleure compagnie pour leur mari... Y pourvoient quelques congrégations féminines, un précepteur parfois, la fondation de Saint-Cyr pour les jeunes filles nobles sans ressources. L'accès des filles à l'enseignement primaire sera plus tardif que celui des garçons. L'enseignement secondaire public pour les jeunes filles ne sera créé par Camille Sée qu'en 1880, sans latin, ce qui les exclut du baccalauréat. La non mixité demeurera de règle pour ne disparaître qu'après mai 1968.

Arrivés au XXI ${ }^{\mathrm{e}}$ siècle, les auteurs présentent notre "système éducatif » comme le résultat d'une totale uniformisation aboutie: filles et garçons de toutes catégories sociales sont scolarisés ensemble à l'école puis au collège, les formations au lycée générales, technologiques et professionnelles - se déroulent en trois ans et débouchent sur le baccalauréat, les professeurs sont formés à bac +5 , quel que soit le type d'enseignement, etc. Le processus centripète est à son terme. Mais la demande d'individualisation va croissant, le succès des officines de soutien scolaire et de coaching en témoigne. Il faudra évoluer...

Que faire? Chacun des auteurs se prononce. L'un propose d'agir par petites touches, en nuances, en poursuivant la décentralisation, la déconcentration et l'autonomie des établissements. Il appelle à une diversification du corps enseignant, comme à une diversification des pratiques pédagogiques. Et surtout, il recommande d'éviter les débats stériles: connaissances/compétences, qualité/quantité, républicains/ 
pédagogues. Il incite à une "pédagogie de la bienveillance", qui croit en la perfectibilité des humains et s'attache à leur réussite.

11 L'autre dénonce le rejet des héritages qui a provoqué deux catastrophes : une piètre maîtrise du français et la montée de l'incivilité ; selon lui, un constructivisme mal compris a fait croire que l'enfant construit seul ses savoirs sans rien attendre de ses aînés ni de la tradition; une " pédagogie de l'hameçon » a prôné que l'envie et le plaisir d'apprendre sont préalables à tout effort, alors que le travail précède l'intérêt ; enfin, le « jeunisme » ambiant a dévalorisé le monde adulte et, avec lui, la trajectoire y menant. Il propose donc de revaloriser la pédagogie du travail, de former l'esprit civique et de faire ainsi accepter l'autorité, et de repenser fondamentalement un projet éducatif pour nos enfants.

12 Au terme d'une lecture passionnante, de retrouvailles stimulantes avec tant de grandes figures de notre histoire, de plongées dans des débats toujours vifs, cette dernière partie, consensuelle et prudente, reflète sans doute la sagesse que confère, avec une analyse sans concession de notre société, une longue expérience des structures et des acteurs de l'École.

\section{AUTEUR}

\section{ANNE-MARIE BARDI}

Anne-Marie Bardi est inspectrice générale honoraire de l'éducation nationale (France). Ancienne élève de l'École normale supérieure de Fontenay-aux-Roses, agrégée de mathématiques, elle a été professeur puis inspectrice d'académie-inspectrice pédagogique régionale de mathématiques. Elle s'est notamment beaucoup impliquée dans le développement de l'usage des technologies de l'information et de la communication dans l'enseignement en France et à l'étranger. annemarie.bardi@orange.fr 